Témoigner Témoigner. Entre histoire et mémoire

Getuigen Revue pluridisciplinaire de la Fondation Auschwitz

$123 \mid 2016$

Traduire le témoignage

\title{
Jasenovac : une cicatrice sur le sol croate
}

Jasenovac: een litteken in de kroatische bodem

\section{Frédéric Crahay}

\section{(2) OpenEdition}

\section{Journals}

\section{Édition électronique}

URL : https://journals.openedition.org/temoigner/5477

DOI : 10.4000/temoigner.5477

ISSN : 2506-6390

\section{Éditeur :}

Éditions du Centre d'études et de documentation Mémoire d'Auschwitz, Éditions Kimé

\section{Édition imprimée}

Date de publication : 1 octobre 2016

Pagination : 197-203

ISBN : 987 2-9600926-4-6

ISSN : 2031-4183

\section{Référence électronique}

Frédéric Crahay, « Jasenovac : une cicatrice sur le sol croate », Témoigner. Entre histoire et mémoire [En ligne], 123 | 2016, mis en ligne le 02 novembre 2021, consulté le 04 novembre 2021. URL : http:// journals.openedition.org/temoigner/5477 ; DOI : https://doi.org/10.4000/temoigner.5477 


\section{$\checkmark$ Site mémoriel}

\section{JASENOVAC \\ UNE CICATRICE SUR LE SOL CROATE}

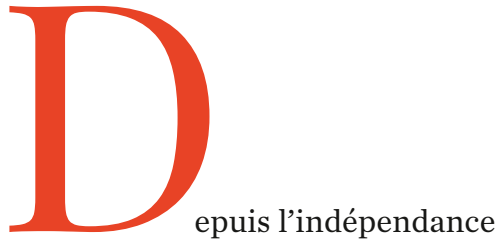
de la République de Croatie en 1991, le site de Jasenovac constitue un lieu de mémoire qui ne cadre plus avec l'image que veut montrer ce jeune État qui cherche à s'affirmer, voire à se défendre contre ses agresseurs. Le lieu a été visité entre 1945 et 1980 par tous les élèves de la nation yougoslave tel qu'il se présentait sous le régime du maréchal Josip Broz Tito et, chaque année, plus de 300000 personnes arpentaient la morne plaine qu'occupe ce site aménagé dans l'immédiat après-guerre. Depuis la disparition de Tito en 1980, la visite n'est plus obligatoire dans le cadre scolaire et, de nos jours, seuls •.•
4 Jasenovac peu après la libération en 1945. On distingue les restes du four de la briqueterie à Jasenovac III.

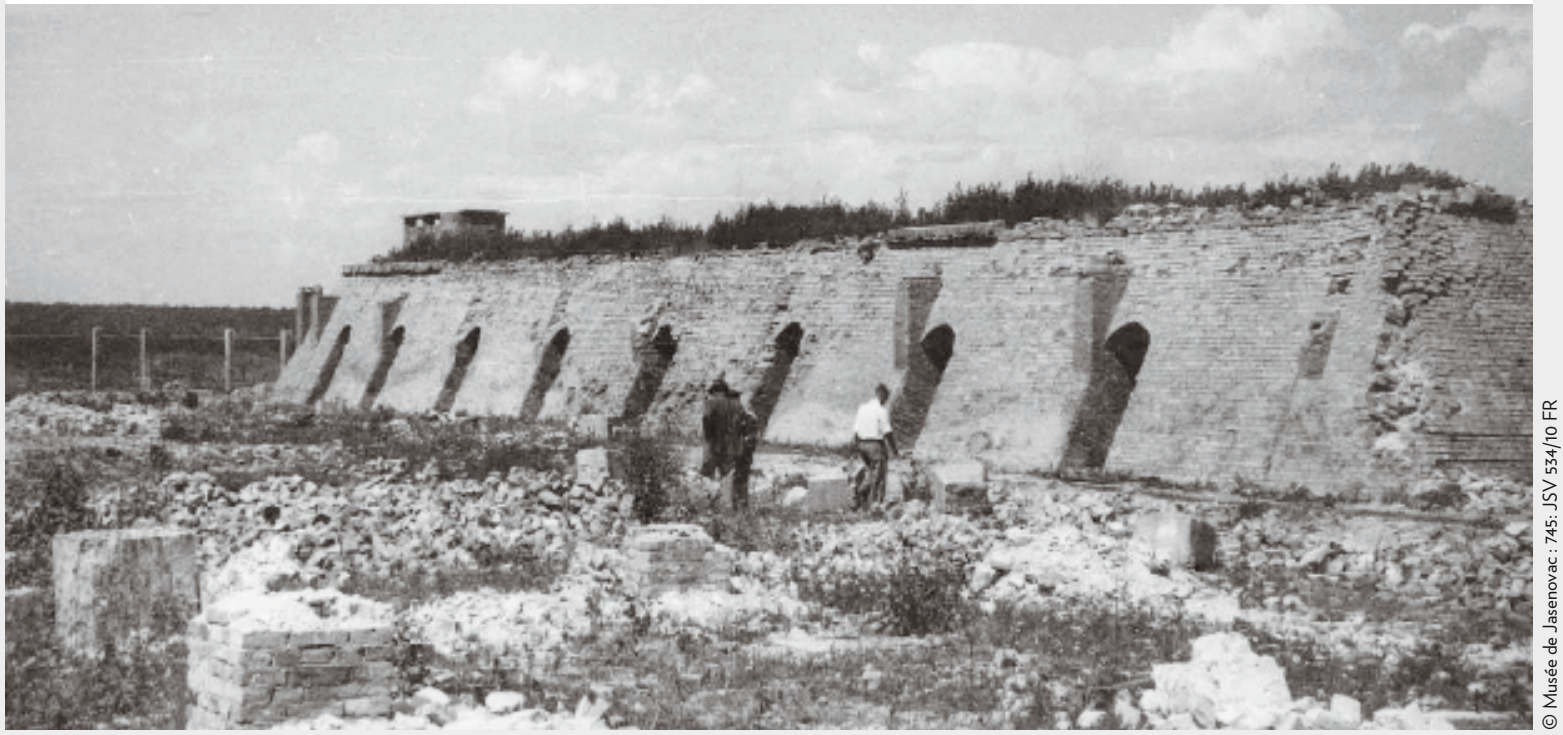


$\cdots 11000$ visiteurs se rendent encore tous les ans à Jasenovac, pour l'essentiel des Serbes, des Bosniaques et des représentants des communautés juive et tsigane présen tes les pays de lex-Yougosite mém sil majeur connarse une telle déchéance?

$\checkmark$ Des tumuli se dressent à présent sur la plaine de Jasenovac III et symbolisent les bâtiments qui s'
dressaient jadis. Le camp de concentration de Jasenovac fut ouvert en août 1941 par desnaris était camps : Krapje (Jasenovac I), Bročica (Jasenovac II), Ciglana (Jasenovac III, (Jasenovac II), Ciglana (Jasenovac III, (Jozar (Jactuellement le mémorial), (Jasen (Jas V) Le IV) et Stara Gradisk (Jasenovac V).Le camp principal (Jasecric terie les annés 19́ en Levillage de Jasenovac fut nettoyé ethniquement de sa populavion serbe qui y avait vécu jusque-là devint durint les ańes. Jasenowac devint durant les cunnes de guerre un paradigme de lhorreur exercee par

indépendant de Croatie (Nezavisna
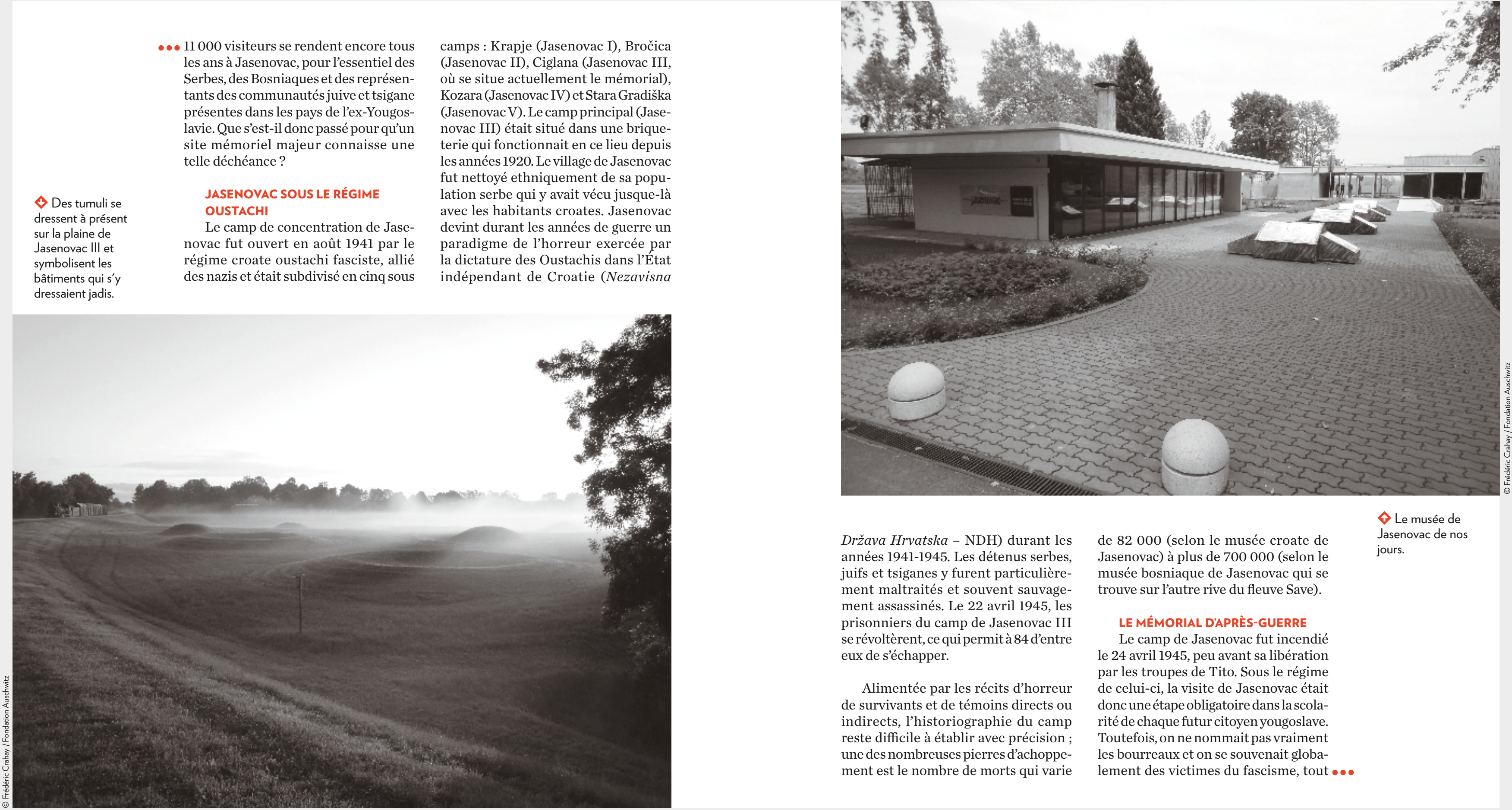

Država Hrvatska - NDH) durant les années 1941-1945. Les détenus serbes, juifs et tsiganes y furent particulièrement maltraites et souvent sauvageprisossassinés. Le 22 avil 1945, les serévins du canp de Jasenovac III eux de s'échapper.

Alimentée par les récits d'horreur de survivants et de témoins directs ou indirects, l'historiographie du camp reste difficile à établir avec précision

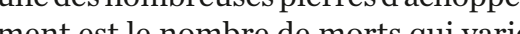

82000 (selon le musée croate de Jasenovac) à plus de 700000 (selon le musée bosniaque de Jasenovac qui se trouve sur l'autre rive du fleuve Save).

\section{LE MÉMORIAL D'APRÈS-GUERRE}

Le camp de Jasenovac fut incendié le 24 avril 1945 , penovac fut incendie par les troupes de Tito. Sous le rén de celui-ci, la visite de Jous le récime doncunétape obligatire dan itéde cha fur tur citoyen you goslave Toutefois, on ne nommait yougare. les bourreaux et on se souv vraiment lement des victimes du fascise tou $\uparrow$ Le musée de 


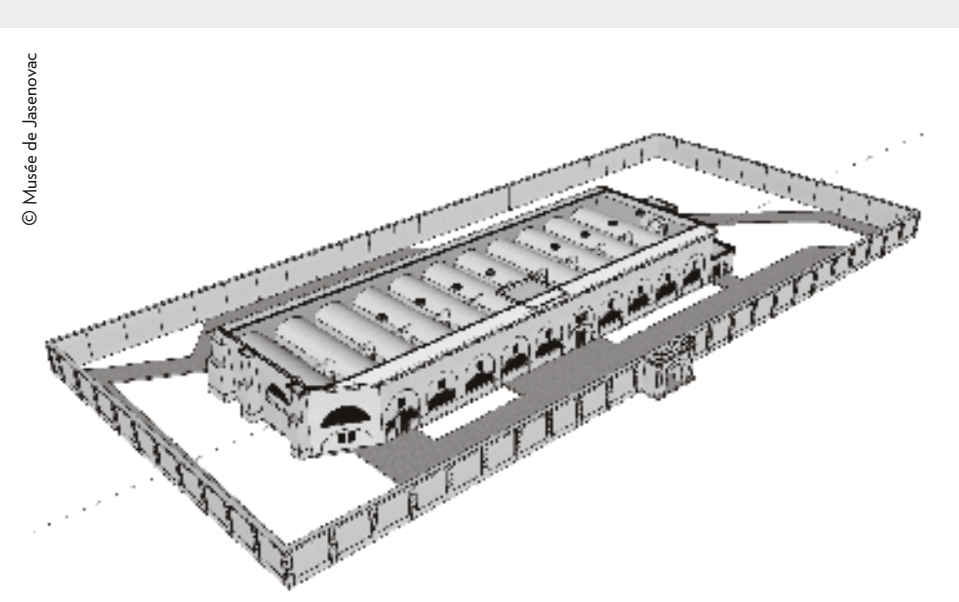

4 Image en 3D

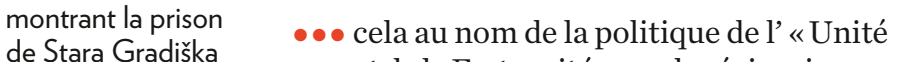
(Jasenovac V)

rénovée. et de la Fraternité» que le régime imposait afin décraser les velleités de séparation entre les peuples yougoslaves et de nuseler les appels revanchards. de la vecing ans dattente après la fin de la Seconde Guere pressionconstantén mémorialles desvicthes pour qu un ménonial voie jour depuis symbolise le site méton qui une syre de l'artiste Bogdaro une œuvre de lartiste Bogdan Bogdanovic et représente une fleur qui doit selon l'es dises de son auteur, «suggérer filé de depasser la souffrance et la folie.» Quelcues annés plus tard, ousere acconpagnat ouvert à son tour.

La guerre de 1991 entre la Croatie et la Serbie changea la donne. De vioet la Serbie changea la donne. De violents antontements eurent lieu dans les alentours du vilage de Jasenovac et, encore aujourd'hui, bon nombre Le muśé de Jasentes stigé Le nusé de Jasenovac fut vide de son contenu par les forces serbes et les docun la ville bo chinu se retrouvent Ils ne seront renis quapiè diapres négociations et l'intervention du United States Holocaust Memorial Museum de Washington, les autorís bosnigues Waulant son, les autorités bosniaques profiterasurt pas pour détres n'en profiteraient pas pour détruire des Oustanis conont sum les deux parties du musée, quireliait pes de parties du musec, ne survécut pas à la guerre. Depuis, les recherches poursuivent séparément Jasenovac se Serbie et en Croatie.

LA PLACE DE JASENOVAC DANS LA CROATIE D'AUJOURD'HUI

La guerre de 1991 que les Croates appellent Homeland War (Guerre patriotique) et qui a été vue du côté croate comme une agression d'un État contre un autre État souverain a, d'une part, ravivé des tensions qui existaien depuis 1945 et, dautre part, infligè de sont pas blessures qui à ce jour ne

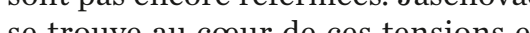
symbolise plus que jams tensions et douboure plus que janais un passe que Janx qui nasse pas. Mlest clair jue Jasenovac gene la Croatie d'aujourd hui qui mise plus volontiers sur le symbolefederateur (pour les Croates) le Danube. Ce lest velle qui se trouve sur de Danube à lest de la Croatie, assiege durant des mois en 1991 par les force serbes, fait figure de ville martyre et symbolise la souffance etlhéroisme. Le role que jouit Jasenovac dans la YouVukovie de Tito a des lors été repris par Kolind ans la Croatie de la présidente Kolinda Grabar-Kitarović. La différence fondamentale est que Jasenovac symla population crinineld une partie de Guerre tie morre mondiale, un passé que la Croatie moderne essaie de moins mettre en var, lieu où les Croates endossent le statut de victimes. Lesélèves croates ont de nos jours largement déserté Jasenovac pour Vukovar, afin d'y suivre une fora paix

Un autre site mémoriel croate contraste avec la mémoire de Jasenocontraste avec la mén séroula le 1 sacre de Blibur-de

nombreux Croates en fuite y furent tués par l’Armée populaire yougoslave de Tito Ce petit village sur a frontire

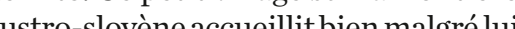
óné par des exićs crutes - cette cotenué prón vie, car étant interdite en Yougoslavie, car ce crime de guerre constituait ituel nation depis l'indép vers un de la Croatie en 1991. Depuis, de plus

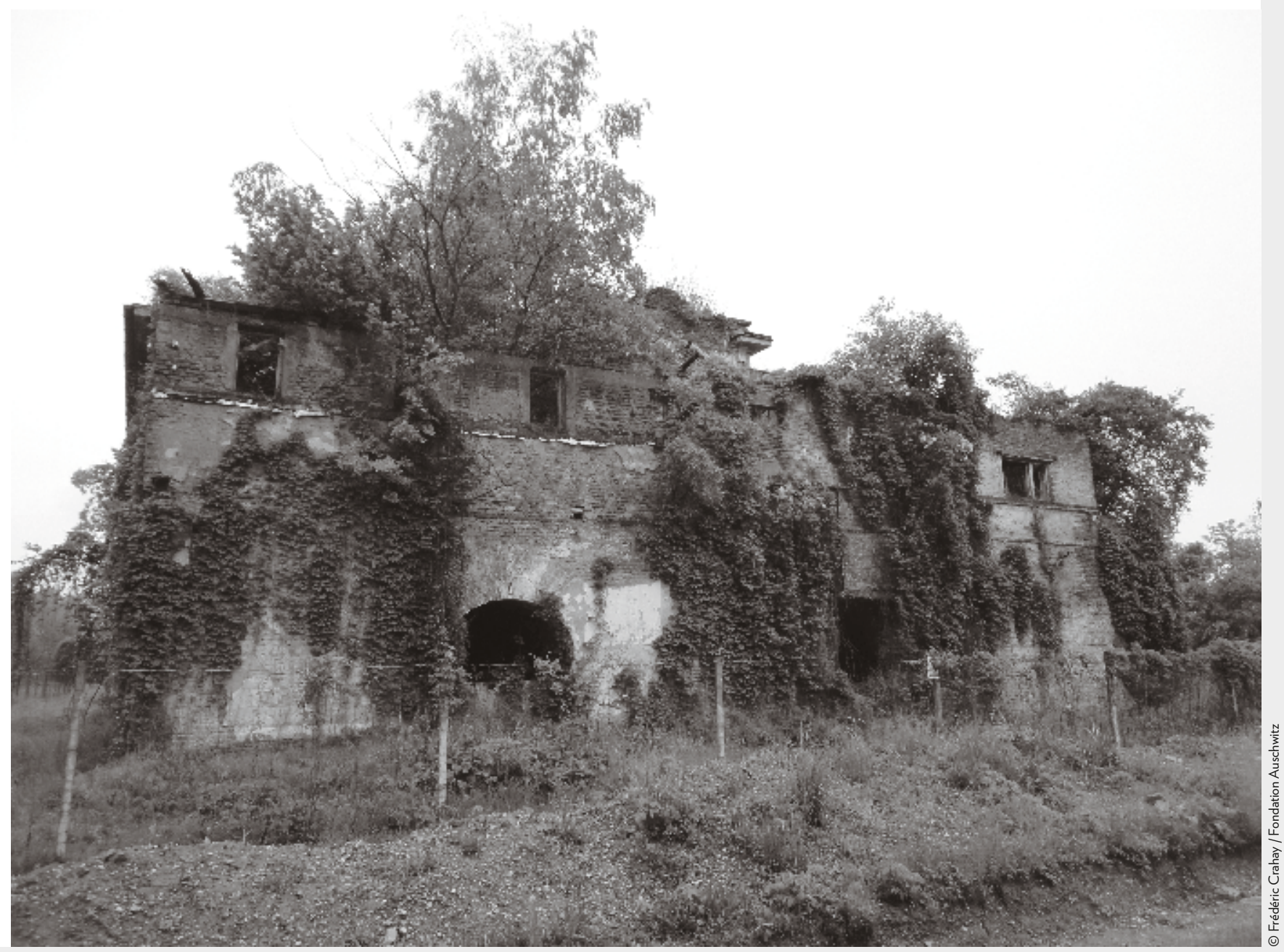


$\bullet$ en plus de politiques liés au gouver nement croate sont présents a la comBleiburg Parmi eux le boullat ble món de la Cultuce, considé sténmistre do sursibie par nom

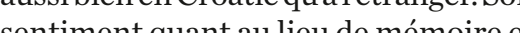
d'histore que contitue Jasenove très net : il fustige la volonté prôné

Bogdanovic

se dresse sur

l'ancienne place

d'appel du camp

de concentration durant les années yougoslaves d'ériger Jasenovac en tant que symbole de Jasenovac en tant que symbole de tique du gouvere le fascisme. La polipare du gourerne lisige pamokratska Zajednica-Union démoDera cratique croate) et la recrudescence des dymos oustachis qui réapparaissent indiffér rues de Croatie ne laissent pas mémer les victimes du rég com-

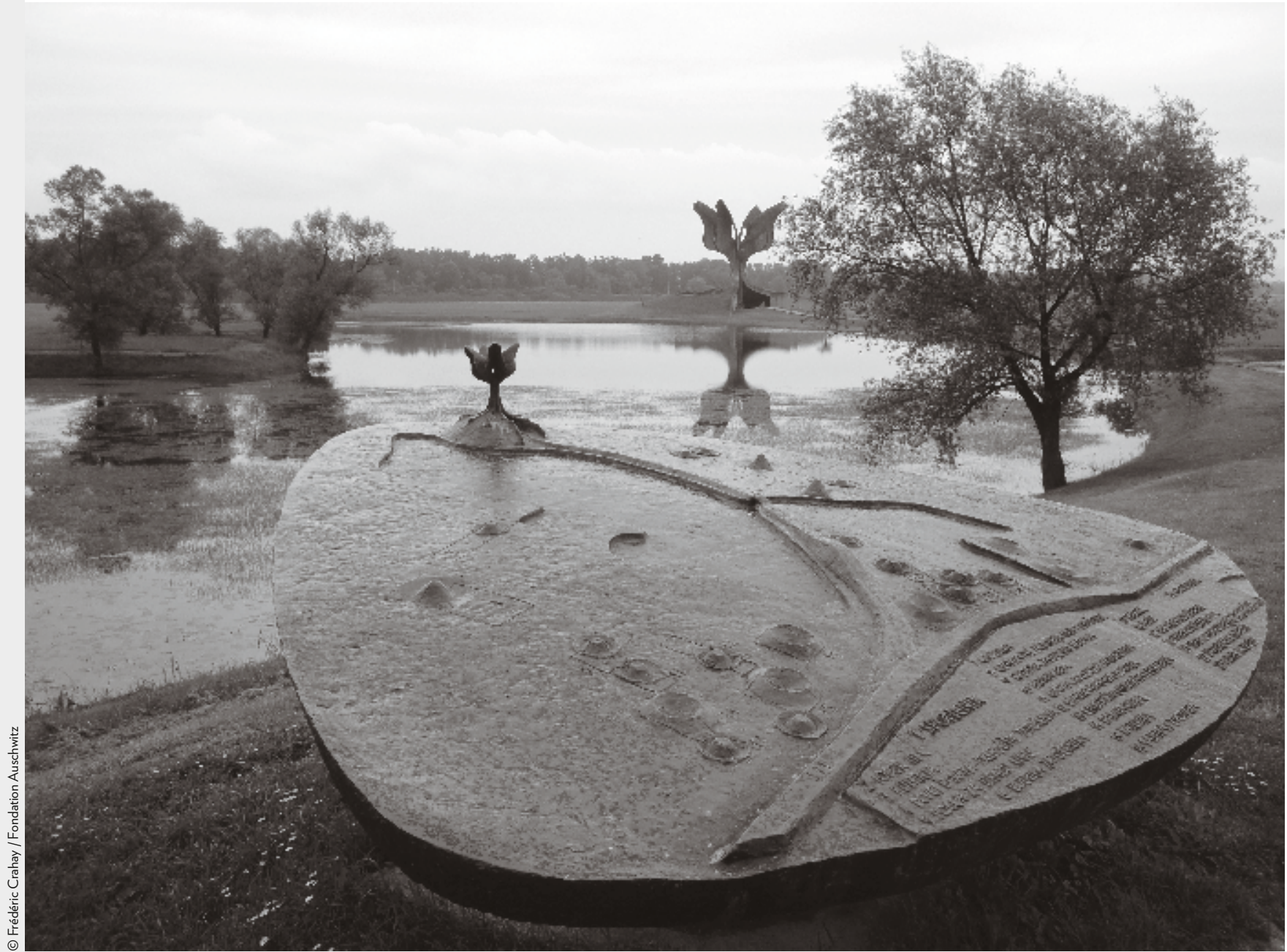

ciste de la Seconde Guerre mondiale. La commémoration officielle de Jase- qui se déroule le 22 avril, da de révolte du camp - ne s'est pas bie passée 2016. En guise de protestation contre la politique du gorvernem. ert tigas associations serbes, juives et tsigás ont préécé vue par l'État croate.

Pour le musée de Jasenovac, labaisse de fréquentation a également eu d'inévitables conséquences économiques, sites. Un exemple qui reflète l'uren des dites. Un exenple qui reflète Turgence Jesta de Stara Gradiska (Jasenovac V), situé femmes et dilos enfants d'orecer juive et tsigane étai dorigine serbe, juive et tsigane étaient enfermés. La gestion de laprison-datant de lépoque austro-hongroise - se faisait depuis Jasenovacll.De nos jours, lebâtiment principal subsiste, mais dans un état ce restron restauration urgente. Les fonds pource faire manquent toutefois etlemusee ne peut que constater lo

\section{CONCLUSION}

Le mémorial de Jasenovac est à un tournant de son existence. La recherche scientifique progresse parmi les vague politiques dont elle dépend souvent Jaur sonfinancement, Cestaussile cas à Jasenova dans lecontextescientique croate. La guerre en ex-Yougoslavie plexifiéla donne

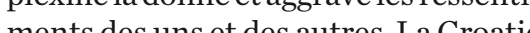
pents les un

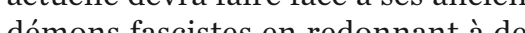
lieur symptostes tiquedonnant à des novac la place qu'ils méritent dans la mémoire collective. Peu de lieux liés à la Seconde Guerre miver sont ions devrout d'asord trouver, des solution

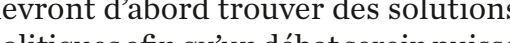
sinstalle safin qu un débat serein puisse historiographique.

Frédéric Crahay

$\rightarrow$ Bibliographie

Egon Berger, (2015) : Jasenovac. Un camp de la mort en Croatie, Genève,
Editions des Syrtes $\diamond$ Jean-Baptiste Chastand : « Mémoire en terrain miné » in : Le Monde, 4 juin 2016.

$\Delta$ Vladimir Dedijer (1992) [1988] : The Yugoslav Auschwitz and the Vatican, reiburg, Ahriman-Verlag.

$\widehat{N}$ Nataša Jovičić (et al.) (2006) : Jasenovac Memorial Site, Jasenovac,

$\Leftrightarrow$ Maja Kućan (2014) : "Izidovi imaju uši" Letters from prisoners of Jasenovac $\triangle$ Nată̌ Mataušc Jasenovac $\diamond$ Nataša Mataušic (2008): Jasenovac, fotomonografija, Zagreb, Jasenovac Memorial Site.

\section{$\rightarrow$ Sitographie}

http: //www.jasenovac.org: le site du Jasenovac Research Institute, situé aux
Etats-Unis, le point de vue de l'Institute est celui des victimes serbes, consulté te $\diamond$ http://www.jusp-jasenovac.hr: le site officiel du Jasenovac Memorial site, le musée du Mémorial, consulté le 9 août 2016. 